\title{
Pattern avoidance in inversion sequences
}

\author{
TOUFIK MANSOUR \\ Department of Mathematics \\ University of Haifa \\ 31905 Haifa, Israel \\ email: toufik@math.haifa.ac.il \\ and \\ MARK SHATTUCK \\ Department of Mathematics \\ University of Tennessee \\ Knoxville, TN 37996 USA \\ email: shattuck@math.utk.edu
}

(Received: February 8, 2015, and in revised form June 22, 2015.)

\begin{abstract}
A permutation of length $n$ may be represented, equivalently, by a sequence $a_{1} a_{2} \cdots a_{n}$ satisfying $0 \leq a_{i}<i$ for all $i$, which is called an inversion sequence. In analogy to the usual case for permutations, the pattern avoidance question is addressed for inversion sequences. In particular, explicit formulas and/or generating functions are derived which count the inversion sequences of a given length that avoid a single pattern of length three. Among the sequences encountered are the Fibonacci numbers, the Schröder numbers, and entry A200753 in OEIS. We make use of both algebraic and combinatorial methods to establish our results. An explicit bijection is given between two of the avoidance classes, and in three cases, the kernel method is used to solve a functional equation satisfied by the generating function enumerating the class in question.
\end{abstract}

Mathematics Subject Classification(2010). 05A15, 05A05.

Keywords: pattern avoidance, permutation, inversion sequence, kernel method.

\section{Introduction}

The pattern avoidance problem for permutations has been extensively studied from various perspectives in both enumerative and algebraic combinatorics; see, e.g., [5] and the references contained therein. The comparable problem has also been considered on other discrete structures such as words, compositions, and set partitions. Suppose a permutation $\sigma$ of $[n]=\{1,2, \ldots, n\}$ is represented as a word $\sigma=$ $\sigma_{1} \sigma_{2} \cdots \sigma_{n}$ wherein $\sigma(i)=\sigma_{i}$ for each $i$. A permutation $\tau$ of shorter length is often referred to as a pattern. In the usual setting, the permutation $\sigma$ is said to contain the pattern $\tau$ if it has a subsequence that is order isomorphic to $\tau$. Otherwise, it is said that $\sigma$ avoids $\tau$. Knuth [6] showed that for any $\tau \in \mathcal{S}_{3}$, the number of permutations of length $n$ that avoid $\tau$ is given by the Catalan number $C_{n}$. Results concerning the avoidance of a subset of $\mathcal{S}_{3}$ were later established by Simion and Schmidt [10].

Another notion of pattern avoidance for permutations involves first writing a permutation as a product of disjoint cycles, then erasing the brackets enclosing the cycles, and finally considering the

DOI: $10.1515 /$ puma-2015-0016 
question of avoidance on the resulting word. See [8], where results concerning this type of avoidance are derived for any subset of $\mathcal{S}_{3}$. The notion is similar to one initially introduced by Callan [2] on set partitions wherein blocks are ordered canonically (according to the size of smallest elements) and brackets are erased giving rise to a permutation on which the avoidance question can be addressed.

Here, we consider yet another definition of avoidance for permutations using a different representation which we now describe. By an inversion in a permutation $\sigma=\sigma_{1} \sigma_{2} \cdots \sigma_{n}$, we will mean an ordered pair $(r, s)$ such that $1 \leq r<s \leq n$ and $\sigma_{r}>\sigma_{s}$. Given $i \in[n]$, let $a_{i}$ denote the number of entries $j$ of $\sigma$ to the right of $i$ such that $j<i$. The word $s=a_{1} a_{2} \cdots a_{n}$ is called the inversion table of the permutation $\sigma$ (see, e.g., [12, p. 21]). Note that $0 \leq a_{i}<i$ for all $i$ and that the total number of inversions of $\sigma$ is $\sum_{i=1}^{n} a_{i}$. Given the inversion table $s$, one can reconstruct the permutation $\sigma$ by initially writing the number 1 and subsequently inserting $i=2,3, \ldots, n$ so that exactly $a_{i}$ numbers smaller than $i$ occur to the right of $i$. Thus, forming the inversion table defines a one-to-one correspondence between permutations of length $n$ and sequences $a_{1} a_{2} \cdots a_{n}$ such that $0 \leq a_{i}<i$ for all $i$, which are known as inversion sequences (see, e.g., [9]).

In this paper, we consider the problem of pattern avoidance on permutations, represented as inversion sequences. In particular, we address the question of avoiding a single permutation pattern of length three by inversion sequences. Here, we will represent the usual patterns by non-negative instead of positive integers. For example, the inversion sequence $s=01031461$ avoids the pattern 201 but has three occurrences of 120, namely, the subsequences 341, 361 and 461 (note that subsequences may or may not contain consecutive numbers). On the other hand, the corresponding permutation $\sigma=74621583$ contains twelve occurrences of the pattern 312 and eight occurrences of 231 . In what follows, we will represent the set of inversion sequences of length $n$ which avoid the pattern $\tau$ by $I \mathcal{S}_{n}(\tau)$.

In the subsequent sections, we will determine explicit formulas and/or generating functions for the number of inversion sequences avoiding any pattern $\tau \in \mathcal{S}_{3}$ in all but one case. Among the sequences encountered are the odd-indexed Fibonacci numbers, the (large) Schröder numbers, and entry A200753 of [11. An explicit bijection is given showing the equivalence of avoiding 201 and 210. Our results are related to recent work of Chen et al. 3] concerning the distribution of ascents on inversion sequences.

For three of the patterns, to find $\left|I \mathcal{S}_{n}(\tau)\right|$, we first refine this number by describing two statistics on $I \mathcal{S}_{n}(\tau)$, and letting $u_{n, m, c}$ denote the number of members of $I \mathcal{S}_{n}(\tau)$ for which the values of the pair of statistics are $m$ and $c$. We write a recurrence for $u_{n, m, c}$, which we convert into functional equation form. We then make use of the kernel method [4] to solve the functional equation and thereby determine $\left|I \mathcal{S}_{n}(\tau)\right|$. Finding appropriate statistics on $I \mathcal{S}_{n}(\tau)$ for which the above strategy could be implemented often was not an easy step and depended on $\tau$. We refer the reader to a paper of Zeilberger [13] for a further description and examples of this strategy of refinement in finding an explicit formula for an unknown sequence.

\section{The case 012}

Let $F_{n}$ denote the Fibonacci number having recurrence

$$
F_{n}=F_{n-1}+F_{n-2}, \quad n \geq 2,
$$

with the initial values $F_{0}=0$ and $F_{1}=1$.

Theorem 2.1 If $n \geq 1$, then $\left|I_{\mathcal{S}_{n}}(012)\right|=F_{2 n-1}$. 
Proof. If $n \geq 2$, then let $A_{n}$ denote the number of 012-avoiding inversion sequences not consisting of all zeros. One can determine a formula for $A_{n}$ as follows by considering the position $i$ of the first non-zero number $j$ within $\pi \in I \mathcal{S}_{n}(012)$, where $1 \leq j<i \leq n$. Note that all non-zero numbers to the right of position $i$ must form a weakly decreasing sequence in $[j]$, for otherwise there would be an occurrence of 012. Suppose that there are exactly $k$ zeros to the right of position $i$ within an enumerated sequence $\pi$. Then there are $\left(\begin{array}{c}n-i \\ k\end{array}\right)$ choices for the positions of these zeros, and once those positions have been decided upon, $\left(\begin{array}{c}n-i-k+j-1 \\ j-1\end{array}\right)$ choices for the non-zero letters to the right of the $i$-th position (as they would correspond to non-negative integer solutions of the equation $x_{1}+x_{2}+\cdots+x_{j}=n-i-k$ ). Since the first $i-1$ letters must all be zero, it follows that

$$
A_{n}=\sum_{i=2}^{n} \sum_{j=1}^{i-1} \sum_{k=0}^{n-i}\left(\begin{array}{c}
n-i \\
k
\end{array}\right)\left(\begin{array}{c}
n-i-k+j-1 \\
j-1
\end{array}\right), \quad n \geq 2
$$

Note that if $2 \leq i \leq n$, then

$$
\begin{aligned}
\sum_{j=1}^{i-1} \sum_{k=0}^{n-i}\left(\begin{array}{c}
n-i \\
k
\end{array}\right)\left(\begin{array}{c}
n-i-k+j-1 \\
j-1
\end{array}\right) & =\sum_{k=0}^{n-i}\left(\begin{array}{c}
n-i \\
k
\end{array}\right) \sum_{j=1}^{i-1}\left(\begin{array}{c}
n-i-k+j-1 \\
n-i-k
\end{array}\right) \\
& =\sum_{k=0}^{n-i}\left(\begin{array}{c}
n-i \\
k
\end{array}\right)\left(\begin{array}{c}
n-k-1 \\
n-i-k+1
\end{array}\right)
\end{aligned}
$$

by virtue of the identity $\sum_{r=m}^{n}\left(\begin{array}{c}r \\ m\end{array}\right)=\left(\begin{array}{c}n+1 \\ m+1\end{array}\right)$, which implies

$$
A_{n}=\sum_{i=2}^{n} \sum_{k=0}^{n-i}\left(\begin{array}{c}
n-i \\
k
\end{array}\right)\left(\begin{array}{c}
n-k-1 \\
n-i-k+1
\end{array}\right), \quad n \geq 2
$$

To determine $A_{n}$, we now compute its generating function:

$$
\begin{aligned}
\sum_{n \geq 2} A_{n} x^{n} & =\sum_{n \geq 2} x^{n} \sum_{i=2}^{n} \sum_{k=0}^{n-i}\left(\begin{array}{c}
n-i \\
k
\end{array}\right)\left(\begin{array}{c}
n-k-1 \\
n-i-k+1
\end{array}\right)=\sum_{n \geq 2} x^{n} \sum_{i=2}^{n} \sum_{\ell=0}^{n-i}\left(\begin{array}{c}
n-i \\
\ell
\end{array}\right)\left(\begin{array}{c}
i+\ell-1 \\
\ell+1
\end{array}\right) \\
& =\sum_{i \geq 2} \sum_{\ell \geq 0}\left(\begin{array}{c}
i+\ell-1 \\
\ell+1
\end{array}\right) \sum_{n \geq i+\ell}\left(\begin{array}{c}
n-i \\
\ell
\end{array}\right) x^{n}=\sum_{i \geq 2} \sum_{\ell \geq 0}\left(\begin{array}{c}
i+\ell-1 \\
\ell+1
\end{array}\right) \frac{x^{i+\ell}}{(1-x)^{\ell+1}} \\
& =\sum_{\ell \geq 0} \frac{x}{(1-x)^{\ell+1}} \sum_{i \geq 2}\left(\begin{array}{c}
i+\ell-1 \\
\ell+1
\end{array}\right) x^{i+\ell-1}=\sum_{\ell \geq 0} \frac{x^{\ell+2}}{(1-x)^{2 \ell+3}} \\
& =\frac{x^{2}}{(1-x)\left(1-3 x+x^{2}\right)} .
\end{aligned}
$$


Thus, we have

$$
\begin{aligned}
\sum_{n \geq 1}\left|I \mathcal{S}_{n}(012)\right| x^{n} & =\frac{x}{1-x}+\sum_{n \geq 2} A_{n} x^{n}=\frac{x}{1-x}+\frac{x^{2}}{(1-x)\left(1-3 x+x^{2}\right)} \\
& =\frac{x(1-x)}{1-3 x+x^{2}}=\sum_{n \geq 1} F_{2 n-1} x^{n}
\end{aligned}
$$

by the generating function formula for the sequence $F_{2 n-1}$ (see, e.g., A001519 in [11]), which completes the proof.

\section{The kernel method and restricted inversion sequences}

For the patterns featured in this section, we make use of the kernel method to determine an explicit formula for the generating function enumerating members of the avoidance class in question.

\subsection{The case 021}

In order to determine its cardinality, we first refine the set consisting of 021-avoiding inversion sequences of length $n$ as follows. Let $\mathcal{A}_{n, m, \ell}$ denote the set of 021-avoiding inversion sequences of length $n$ in which the largest letter is $m$ and the last letter is $\ell$. Note that $I \mathcal{S}_{n}(021)=\cup_{m, \ell} \mathcal{A}_{n, m, \ell}$.

Let $a_{n, m, \ell}=\left|\mathcal{A}_{n, m, \ell}\right|$ if $0 \leq \ell \leq m<n$, and put $a_{n, m, \ell}=0$ otherwise. For example, if $n=5$, $m=2$, and $\ell=0$, then $a_{5,2,0}=8$, the enumerated sequences being $0 x 020,0 x 120,0 x 200$, and $0 x 220$, where $x=0,1$. The array of numbers $a_{n, m, \ell}$ may be determined recursively as follows.

LEMMA 3.1 If $n \geq 2$, then

$$
a_{n, m, 0}=\sum_{j=0}^{m} a_{n-1, m, j}, \quad 0 \leq m \leq n-1,
$$

and

$$
a_{n, m, m}=\sum_{i=0}^{m} \sum_{j=0}^{i} a_{n-1, i, j}, \quad 1 \leq m \leq n-1,
$$

with $a_{n, m, \ell}=0$ if $1 \leq \ell<m<n$ and $a_{1,0,0}=1$.

Proof. The last two statements follow easily from the definitions since an inversion sequence whose final letter is positive and strictly less than the largest letter always contains an occurrence of the pattern 021. Next observe that deletion of the last letter of $\pi \in \mathcal{A}_{n, m, 0}$, which is seen to be extraneous concerning the avoidance of 021 , results in a member of $\mathcal{A}_{n-1, m, j}$ for some $0 \leq j \leq m$, which implies (1). Finally, if $1 \leq m \leq n-1$, then deletion of the last letter of $\pi \in \mathcal{A}_{n, m, m}$ results in a member of $\mathcal{A}_{n-1, i, j}$ for some $0 \leq i \leq m$ and $0 \leq j \leq i$. Summing over $i$ and $j$ gives (2) and completes the proof. 
If $n \geq 1$ and $0 \leq m<n$, then let

$$
a_{n, m}(v)=\sum_{j=0}^{m} a_{n, m, j} v^{j}
$$

If $n \geq 1$, then let

$$
a_{n}(u, v)=\sum_{m=0}^{n-1} a_{n, m}(v) u^{m} .
$$

Define the generating function $f(x ; u, v)$ by

$$
f(x ; u, v)=\sum_{n \geq 1} a_{n}(u, v) x^{n} .
$$

Then $f(x ; u, v)$ satisfies the following functional equation.

LEMMA 3.2 We have

$$
f(x ; u, v)=\frac{x-2 x^{2}}{1-x}+x f(x ; u, 1)+\frac{x}{1-u v} f(x ; u v, 1)-\frac{x u v}{1-u v} f(x u v ; 1,1) .
$$

Proof. By recurrences (1) and (2), we have

$$
a_{n, m}(v)=a_{n-1, m}(1)+v^{m} \sum_{i=0}^{m} a_{n-1, i}(1), \quad 1 \leq m<n,
$$

with $a_{n, 0}(v)=a_{n, 0,0}=1$ if $n \geq 1$. Multiplying both sides of (4) by $u^{m}$ and summing over $1 \leq m<n$ gives

$$
\begin{aligned}
a_{n}(u, v) & =1+\sum_{m=1}^{n-1} a_{n-1, m}(1) u^{m}+\sum_{m=1}^{n-1}(u v)^{m} \sum_{i=0}^{m} a_{n-1, i}(1) \\
& =1+\left(-1+a_{n-1}(u, 1)+\left(-1+\sum_{i=0}^{n-1} a_{n-1, i}(1) \sum_{m=i}^{n-1}(u v)^{m}\right)\right. \\
& =-1+a_{n-1}(u, 1)+\sum_{i=0}^{n-1} a_{n-1, i}(1)\left(\frac{(u v)^{i}-(u v)^{n}}{1-u v}\right) \\
& =-1+a_{n-1}(u, 1)+\frac{1}{1-u v} a_{n-1}(u v, 1)-\frac{(u v)^{n}}{1-u v} a_{n-1}(1,1), \quad n \geq 2 .
\end{aligned}
$$

Multiplying both sides of (5) by $x^{n}$ and summing over $n \geq 2$ yields

$$
f(x ; u, v)-x=-\frac{x^{2}}{1-x}+x f(x ; u, 1)+\frac{x}{1-u v} f(x ; u v, 1)-\frac{x u v}{1-u v} f(x u v ; 1,1),
$$

which completes the proof. 
Let $s_{n}$ denote the $n$-th Schröder number. Recall that

$$
\sum_{n \geq 0} s_{n} x^{n}=\frac{1-x-\sqrt{1-6 x+x^{2}}}{2 x} ;
$$

see, e.g., A006318 in [11]. Equivalently, the sequence $s_{n}$ is determined by the recurrence

$$
(n+1) s_{n}=3(2 n-1) s_{n-1}-(n-2) s_{n-2}, \quad n \geq 2,
$$

along with the initial values $s_{0}=1$ and $s_{1}=2$. The next result provides a new combinatorial interpretation of the Schröder numbers.

THEOREM 3.3 If $n \geq 1$, then $\left|I \mathcal{S}_{n}(021)\right|=s_{n-1}$.

Proof. Letting $v=1$ in (3) implies

$$
\left(1-x-\frac{x}{1-u}\right) f(x ; u, 1)=\frac{x-2 x^{2}}{1-x}-\frac{x u}{1-u} f(x u ; 1,1) .
$$

Applying the kernel method (see [4]) and setting $u=\frac{1-2 x}{1-x}$ in (6) cancels out the left-hand side and gives

$$
f\left(\frac{x(1-2 x)}{1-x} ; 1,1\right)=\frac{x}{1-x} .
$$

If $y=\frac{x(1-2 x)}{1-x}$, then solving for $x$ in terms of $y$ gives

$$
x=\frac{1+y-\sqrt{1-6 y+y^{2}}}{4},
$$

where we have chosen the negative root since $f(0 ; 1,1)=0$.

Replacing $\frac{x(1-2 x)}{1-x}$ with $y$ in $(7)$ then yields

$$
\begin{aligned}
\sum_{n \geq 1}\left|I \mathcal{S}_{n}(021)\right| y^{n} & =f(y ; 1,1)=\frac{x}{1-x} \\
& =\frac{1+y-\sqrt{1-6 y+y^{2}}}{3-y+\sqrt{1-6 y+y^{2}}} \\
& =\frac{1-y-\sqrt{1-6 y+y^{2}}}{2} \\
& =\sum_{n \geq 1} s_{n-1} y^{n},
\end{aligned}
$$

which completes the proof. 
Remark. In view of Theorem 3.3, we leave as a challenge to the reader the task of finding a bijection between $I \mathcal{S}_{n}(021)$ and the set of Schröder paths of semilength $n-1$, i.e., the set of lattice paths from the origin to the point $(2 n-2,0)$ using $(1,1),(1,-1)$, and $(2,0)$ steps and never dipping below the $x$-axis.

It is possible to find an explicit formula for the solution to the functional equation (3). Using the expression found in the previous proof for $f(x ; 1,1)$ in $(6)$ yields

$$
f(x ; u, 1)=\frac{x\left(2-4 x-3 u+5 x u+x u^{2}-x^{2} u^{2}\right)}{2(1-x)(1-2 x+(x-1) u)}+\frac{x u \sqrt{1-6 x u+x^{2} u^{2}}}{2(1-2 x+(x-1) u)} .
$$

We observe that the coefficient of $x^{n} u^{m}$ in $f(x ; u, 1)$ is given by sequence A145035 in [11]. Substituting this formula for $f(x ; u, 1)$ into the functional equation (3) shows that it has a unique solution $f(x ; u, v)$ given by

$$
\begin{aligned}
f(x ; u, v)= & \frac{x(1-2 x)\left(2-4 x-2 u+x u+3 x^{2} u+x^{2} u^{2}-x^{3} u^{2}\right)}{2(1-x)(1-2 x+(x-1) u)(1-2 x+(x-1) u v)} \\
& -\frac{x u\left(3-9 x-3 u+5 x u+6 x^{2}+x^{2} u^{2}-x^{3} u^{2}\right) v}{2(1-2 x+(x-1) u)(1-2 x+(x-1) u v)}+\frac{x^{2} u^{2}(1-x) v^{2}}{2(1-2 x+(x-1) u v)} \\
& +\frac{x^{2} u \sqrt{1-6 x u+x^{2} u^{2}}}{2(1-2 x+(x-1) u)}+\frac{x(1-x) u v \sqrt{1-6 x u v+x^{2} u^{2} v^{2}}}{2(1-2 x+(x-1) u v)} .
\end{aligned}
$$

\subsection{The case 102}

To determine the number of 102-avoiding inversion sequences of length $n$, we refine the set consisting of these sequences as follows. If $0 \leq m<\ell \leq n$, then let $\mathcal{B}_{n, m, \ell}$ denote the set of 102-avoiding inversion sequences of length $n$ in which the largest letter is $m$ with the left-most occurrence of $m$ in position $\ell$. Note that $I \mathcal{S}_{n}(102)=\cup_{m, \ell} \mathcal{B}_{n, m, \ell}$.

Let $b_{n, m, \ell}=\left|\mathcal{B}_{n, m, \ell}\right|$ if $0 \leq m<\ell \leq n$, and put $b_{n, m, \ell}=0$ otherwise. For example, if $n=4$, $m=2$, and $\ell=4$, then $b_{4,2,4}=3$, the enumerated sequences being 0002, 0012, and 0112. The array of numbers $b_{n, m, \ell}$ may be defined recursively as follows.

Lemma 3.4 If $1 \leq m<\ell \leq n$, then

$$
b_{n, m, \ell}=\sum_{i=\ell}^{n-1} b_{n-1, m, i}+\sum_{j=0}^{m-1} \sum_{i=\ell-1}^{n-1} b_{n-1, j, i}+\sum_{j=0}^{m-1} \sum_{i=1}^{\ell-2} b_{n-i-1, j, \ell-i-1},
$$

with $b_{n, 0, \ell}=\delta_{\ell, 1}$ for $1 \leq \ell \leq n$.

Proof. The initial condition when $m=0$ follows easily from the definitions. To show (8), first note that if $m$ occurs more than once within a member of $\mathcal{B}_{n, m, \ell}$, then the left-most $m$ is extraneous concerning avoidance of 102. Deletion of this $m$ then results in a member of $\mathcal{B}_{n-1, m, i}$ for some $\ell \leq i \leq n-1$, which implies that there are $\sum_{i=\ell}^{n-1} b_{n-1, m, i}$ members of $\mathcal{B}_{n, m, \ell}$ where the letter $m$ occurs more than once.

Suppose now that the letter $m$ occurs only once within $\pi \in \mathcal{B}_{n, m, \ell}$ and that the second largest letter of $\pi$ is $j$. First assume that the left-most occurrence of $j$ within $\pi$ is either in position $\ell-1$ (i.e., just to the left of the $m$ ) or in positions $\ell+1$ through $n$, inclusive. In each of these cases, the $m$ is 
extraneous since the first $\ell-2$ letters of $\pi$ are governed by a $j$ either coming just before $m$ or at some point after $m$. Deletion of $m$ from $\pi$ then results in a member of $\mathcal{B}_{n-1, j, i}$ for some $0 \leq j \leq m-1$ and $\ell-1 \leq i \leq n-1$, and summing over these indices gives the second term in (8). On the other hand, if the left-most $j$ of $\pi$ occurs in positions 1 through $\ell-2$, then each letter between the left-most $j$ and $m$ must also be a $j$, by definition of $j$, for otherwise there would be an occurrence of 102 , which is not possible. Therefore, deletion of $m$ as well as all $i$ letters (equal to $j$ ) that occur between the left-most $j$ and $m$ results in a member of $\mathcal{B}_{n-i-1, j, \ell-i-1}$ for some $0 \leq j \leq m-1$ and $1 \leq i \leq \ell-2$. Summing over all $j$ and $i$ gives the third term on the right-hand side of $(8)$ and completes the proof.

If $n \geq 2$ and $2 \leq \ell \leq n$, then let

$$
b_{n, \ell}(u)=\sum_{m=1}^{\ell-1} b_{n, m, \ell} u^{m},
$$

with $b_{n, 1}(u)=0$. If $n \geq 2$, then let

$$
b_{n}(u, v)=\sum_{\ell=2}^{n} b_{n, \ell}(u) v^{\ell},
$$

with $b_{1}(u, v)=0$. Define the generating function $g(x ; u, v)$ by

$$
g(x ; u, v)=\sum_{n \geq 2} b_{n}(u, v) x^{n} .
$$

Then $g(x ; u, v)$ satisfies the following functional equation.

LEMMA 3.5 We have

$$
\begin{aligned}
g(x ; u, v)= & \frac{x^{2} u v^{2}}{(1-x)(1-x v)(1-x u v)}+\frac{x v}{(1-v)(1-u v)} g(x ; u v, 1) \\
& +\frac{x u^{2} v^{2}(1-x)}{(1-u)(1-u v)(1-x u v)} g(x ; 1, u v)+\frac{x v(u-1+x v-u v)}{(1-u)(1-v)(1-x v)} g(x ; u, v) .
\end{aligned}
$$

Proof. Multiplying both sides of $(8)$ by $u^{m} v^{\ell}$ and summing over $1 \leq m<\ell \leq n$ will yield a recurrence satisfied by the polynomials $b_{n}(u, v)$. We first must simplify the following three sums, which come from the first, second, and third terms, respectively, in (8):

$$
\begin{aligned}
\sum_{m=1}^{n-1} u^{m} & \sum_{\ell=m+1}^{n} v^{\ell} \sum_{i=\ell}^{n-1} b_{n-1, m, i}=\sum_{m=1}^{n-1} u^{m} \sum_{i=m+1}^{n-1} b_{n-1, m, i} \sum_{\ell=m+1}^{i} v^{\ell} \\
& =\frac{v}{1-v}\left(\sum_{m=1}^{n-1}(u v)^{m} \sum_{i=m+1}^{n-1} b_{n-1, m, i}-\sum_{m=1}^{n-1} u^{m} \sum_{i=m+1}^{n-1} b_{n-1, m, i} v^{i}\right) \\
& =\frac{v}{1-v}\left(\sum_{i=2}^{n-1} b_{n-1, i}(u v)-\sum_{i=2}^{n-1} b_{n-1, i}(u) v^{i}\right) \\
& =\frac{v}{1-v}\left(b_{n-1}(u v, 1)-b_{n-1}(u, v)\right),
\end{aligned}
$$




$$
\begin{aligned}
& \sum_{\ell=2}^{n} v^{\ell} \sum_{m=1}^{\ell-1} u^{m} \sum_{j=0}^{m-1} \sum_{i=\ell-1}^{n-1} b_{n-1, j, i}=\sum_{\ell=2}^{n} v^{\ell} \sum_{j=0}^{\ell-2} \sum_{i=\ell-1}^{n-1} b_{n-1, j, i} \sum_{m=j+1}^{\ell-1} u^{m} \\
&=\sum_{j=0}^{n-2} \sum_{\ell=j+2}^{n} v^{\ell} \sum_{i=\ell-1}^{n-1} b_{n-1, j, i} \frac{u^{j+1}-u^{\ell}}{1-u} \\
&=\frac{u}{1-u} \sum_{i=1}^{n-1} \sum_{j=0}^{i-1} b_{n-1, j, i} u^{j}\left(\frac{v^{j+2}-v^{i+2}}{1-v}\right) \\
&-\frac{1}{1-u} \sum_{i=1}^{n-1} \sum_{j=0}^{i-1} b_{n-1, j, i}\left(\frac{(u v)^{j+2}-(u v)^{i+2}}{1-u v}\right) \\
&=\frac{u v^{2}}{(1-u)(1-v)}\left(\sum_{i=1}^{n-1}\left(b_{n-1, i}(u v)+\delta_{i, 1}\right)-\sum_{i=1}^{n-1}\left(b_{n-1, i}(u)+\delta_{i, 1}\right) v^{i}\right) \\
&-\frac{u^{2} v^{2}}{(1-u)(1-u v)}\left(\sum_{i=1}^{n-1}\left(b_{n-1, i}(u v)+\delta_{i, 1}\right)-\sum_{i=1}^{n-1}\left(b_{n-1, i}(1)+\delta_{i, 1}\right)(u v)^{i}\right) \\
&= u v^{2}+\frac{u v^{2}}{(1-v)(1-u v)} b_{n-1}(u v, 1)+\frac{u^{2} v^{2}}{(1-u)(1-u v)} b_{n-1}(1, u v) \\
&-\frac{u v^{2}}{(1-u)(1-v)} b_{n-1}(u, v),
\end{aligned}
$$

and

$$
\begin{aligned}
\sum_{\ell=2}^{n} v^{\ell} \sum_{m=1}^{\ell-1} u^{m} \sum_{j=0}^{m-1} \sum_{i=1}^{\ell-2} b_{n-i-1, j, \ell-i-1}=\sum_{\ell=2}^{n} v^{\ell} \sum_{i=1}^{\ell-2} \sum_{j=0}^{\ell-2} b_{n-i-1, j, \ell-i-1} \frac{u^{j+1}-u^{\ell}}{1-u} \\
=\frac{u}{1-u} \sum_{\ell=2}^{n} v^{\ell} \sum_{i=1}^{\ell-2}\left(b_{n-i-1, \ell-i-1}(u)+\delta_{i, \ell-2}\right) \\
\quad-\frac{1}{1-u} \sum_{\ell=2}^{n}(u v)^{\ell} \sum_{i=1}^{\ell-2}\left(b_{n-i-1, \ell-i-1}(1)+\delta_{i, \ell-2}\right) \\
=\frac{u}{1-u} \sum_{i=1}^{n-2} v^{i+1} \sum_{\ell=i+2}^{n}\left(b_{n-i-1, \ell-i-1}(u)+\delta_{\ell, i+2}\right) v^{\ell-i-1} \\
\quad-\frac{1}{1-u} \sum_{i=1}^{n-2}(u v)^{i+1} \sum_{\ell=i+2}^{n}\left(b_{n-i-1, \ell-i-1}(1)+\delta_{\ell, i+2}\right)(u v)^{\ell-i-1} \\
=\frac{u}{1-u} \sum_{i=1}^{n-2}\left(b_{n-i-1}(u, v)+v\right) v^{i+1}-\frac{1}{1-u} \sum_{i=1}^{n-2}\left(b_{n-i-1}(1, u v)+u v\right)(u v)^{i+1}
\end{aligned}
$$


Combining the three sums above then yields the recurrence

$$
\begin{aligned}
b_{n}(u, v)= & u v^{2}+\frac{v}{(1-v)(1-u v)} b_{n-1}(u v, 1)+\frac{u^{2} v^{2}}{(1-u)(1-u v)} b_{n-1}(1, u v) \\
& +\frac{v(u-1-u v)}{(1-u)(1-v)} b_{n-1}(u, v)+\frac{u}{1-u} \sum_{i=1}^{n-2}\left(b_{n-i-1}(u, v)+v\right) v^{i+1} \\
& -\frac{1}{1-u} \sum_{i=1}^{n-2}\left(b_{n-i-1}(1, u v)+u v\right)(u v)^{i+1}, \quad n \geq 2,
\end{aligned}
$$

with $b_{1}(u, v)=0$. Multiplying both sides of 10$)$ by $x^{n}$ and summing over $n \geq 2$ yields

$$
\begin{aligned}
g(x ; u, v)= & \frac{x^{2} u v^{2}}{1-x}+\frac{x v}{(1-v)(1-u v)} g(x ; u v, 1)+\frac{x u^{2} v^{2}}{(1-u)(1-u v)} g(x ; 1, u v) \\
& +\frac{x v(u-1-u v)}{(1-u)(1-v)} g(x ; u, v) \\
& +\frac{x^{2} u v^{2}}{1-u}\left(\frac{1}{1-x v} g(x ; u, v)-\frac{u}{1-x u v} g(x ; 1, u v)+\frac{x v\left(1-u^{2}-x u v+x u^{2} v\right)}{(1-x)(1-x v)(1-x u v)}\right),
\end{aligned}
$$

which gives the desired result.

It is possible to express the functional equation in the prior lemma in a more convenient form.

Lemma 3.6 We have

$$
g(x ; v, 1)=\frac{v^{2}(1-x)(1-w)}{(v-w)(1-x v)} g(x ; 1, v)-\frac{x v(1-v)(1-w)}{(1-x)(1-x v)(1-x w)},
$$

where $w=w(x, v)$ satisfies the cubic equation

$$
\left(x^{2}-x\right) w^{3}+w^{2}-(1+v) w+v=0 .
$$

Proof. We apply the kernel method and set the coefficients of $g(x ; u, v)$ on both sides of equation (9) equal. Solving for $u=u(x, v)$ in terms of $x$ and $v$ gives

$$
u=1+\frac{x v^{2}(x-1)}{v-1} .
$$

Let $\alpha(x, v)$ be defined by

$$
\alpha(x, v)=u v=v+\frac{x v^{3}(x-1)}{v-1},
$$

which we'll denote by $\alpha$. Substituting the expression for $u$ given in 13 into 9 , and simplifying, yields

$$
g(x ; \alpha, 1)=\frac{\alpha^{2}(1-x)(1-v)}{(\alpha-v)(1-x \alpha)} g(x ; 1, \alpha)-\frac{x \alpha(1-\alpha)(1-v)}{(1-x)(1-x \alpha)(1-x v)} .
$$

Note that $\alpha(x, w)=v$, where $w=w(x, v)$ is defined by (12). Replacing $v$ with $w$ on both sides of (14) then gives 11 . 
THEOREM 3.7 The generating function $A(x)=g(x ; 1,1)+\frac{1}{1-x}$ satisfies

$$
A(x)=1+\left(x-x^{2}\right) A(x)^{3} .
$$

Thus, the number of 102-avoiding inversion sequences of length $n$ is given by the formula

$$
\sum_{m=\left\lceil\frac{n}{2}\right\rceil}^{n} \frac{(-1)^{n-m}}{2 m+1}\left(\begin{array}{c}
3 m \\
m
\end{array}\right)\left(\begin{array}{c}
m \\
n-m
\end{array}\right), \quad n \geq 0 .
$$

Proof. Note that $g(x ; 1,1)$ is the generating function which counts all 102-avoiding inversion sequences except those consisting of a (possibly empty) sequence of 0's. Thus, $A(x)$ counts all 102-avoiding inversion sequences of length $n$ for each $n \geq 0$. The second statement is then an immediate consequence of the first and the Lagrange inversion theorem.

To prove the first statement, let $w_{1}, w_{2}$, and $w_{3}$ denote the three roots of the equation (12). Substituting $w_{1}$ and $w_{2}$ into (11) then gives

$$
g(x ; v, 1)=\frac{v^{2}(1-x)\left(1-w_{1}\right)}{\left(v-w_{1}\right)(1-x v)} g(x ; 1, v)-\frac{x v(1-v)\left(1-w_{1}\right)}{(1-x)(1-x v)\left(1-x w_{1}\right)}
$$

and

$$
g(x ; v, 1)=\frac{v^{2}(1-x)\left(1-w_{2}\right)}{\left(v-w_{2}\right)(1-x v)} g(x ; 1, v)-\frac{x v(1-v)\left(1-w_{2}\right)}{(1-x)(1-x v)\left(1-x w_{2}\right)} .
$$

Solving this system of equations in the quantities $g(x ; v, 1)$ and $g(x ; 1, v)$ yields

$$
g(x ; 1, v)=\frac{x\left(w_{1}-v\right)\left(w_{2}-v\right)}{v(x-1)\left(x w_{1}-1\right)\left(x w_{2}-1\right)} .
$$

Taking $v=1$ in 16 gives

$$
g(x ; 1,1)=\frac{x\left(w_{1}-1\right)\left(w_{2}-1\right)}{(x-1)\left(x w_{1}-1\right)\left(x w_{2}-1\right)},
$$

where $w_{1}$ and $w_{2}$ satisfy $x(1-x) w^{3}=(w-1)^{2}$.

Note that

$$
\left(w_{1}-1\right)\left(w_{2}-1\right)=w_{1} w_{2}-\left(w_{1}+w_{2}\right)+1=\frac{1}{w_{3} x(1-x)}-\left(\frac{1}{x(1-x)}-w_{3}\right)+1
$$

and

$$
\left(x w_{1}-1\right)\left(x w_{2}-1\right)=\frac{x}{w_{3}(1-x)}-\left(\frac{1}{1-x}-w_{3} x\right)+1,
$$

since $w_{1}, w_{2}$, and $w_{3}$ are roots of the polynomial equation $x(1-x) w^{3}-w^{2}+2 w-1=0$. Thus, we have

$$
\begin{aligned}
\frac{\left(w_{1}-1\right)\left(w_{2}-1\right)}{\left(x w_{1}-1\right)\left(x w_{2}-1\right)} & =\frac{1-w_{3}+w_{3} x\left(1+w_{3}\right)(1-x)}{x^{2}-w_{3} x+w_{3} x(1-x)+w_{3}^{2} x^{2}(1-x)} \\
& =\frac{w_{3}^{2}\left(1-w_{3}\right)+\left(1-w_{3}\right)^{2}\left(1+w_{3}\right)}{w_{3}^{2} x^{2}-w_{3}^{3} x+\left(1-w_{3}\right)^{2}+w_{3} x\left(1-w_{3}\right)^{2}} \\
& =\frac{1-w_{3}}{(x-1)^{2} w_{3}^{2}+(x-2) w_{3}+1}
\end{aligned}
$$


which implies

$$
\begin{aligned}
A(x) & =g(x ; 1,1)+\frac{1}{1-x}=\frac{x\left(1-w_{3}\right)}{(x-1)\left((x-1)^{2} w_{3}^{2}+(x-2) w_{3}+1\right)}+\frac{1}{1-x} \\
& =\frac{(1-x) w_{3}^{2}-2 w_{3}+1}{(x-1)^{2} w_{3}^{2}+(x-2) w_{3}+1}=\frac{\left(w_{3}-1\right)^{2}-x w_{3}^{2}}{\left(w_{3}-1\right)^{2}+\left(x^{2}-2 x\right) w_{3}^{2}+x w_{3}} \\
& =\frac{x(1-x) w_{3}^{3}-x w_{3}^{2}}{x(1-x) w_{3}^{3}+\left(x^{2}-2 x\right) w_{3}^{2}+x w_{3}}=\frac{(1-x) w_{3}^{2}-w_{3}}{(1-x) w_{3}^{2}+(x-2) w_{3}+1} .
\end{aligned}
$$

We now verify (15) using the expression for $A(x)$ given by (18). Upon clearing fractions, to complete the proof, we need to show

$$
\begin{aligned}
& \left((1-x) w_{3}^{2}+(x-2) w_{3}+1\right)^{3}+x(1-x)\left((1-x) w_{3}^{2}-w_{3}\right)^{3}=\left((1-x) w_{3}^{2}-w_{3}\right)\left((1-x) w_{3}^{2}+(x-2) w_{3}+1\right)^{2}, \\
& \text { i.e., } \\
& \qquad\left((1-x) w_{3}^{2}+(x-2) w_{3}+1\right)^{2}\left((x-1) w_{3}+1\right)=x(x-1)\left((1-x) w_{3}^{2}-w_{3}\right)^{3},
\end{aligned}
$$

or

$$
\left((1-x) w_{3}^{2}+(x-2) w_{3}+1\right)^{2}=x(1-x) w_{3}^{3}\left((x-1) w_{3}+1\right)^{2} .
$$

Since $x(1-x) w_{3}^{3}=\left(1-w_{3}\right)^{2}$, the last equation holds via the factorization

$$
(1-x) w_{3}^{2}+(x-2) w_{3}+1=\left(1-w_{3}\right)\left((x-1) w_{3}+1\right),
$$

which completes the proof.

Remark. The sequence enumerating $I \mathcal{S}_{n}(102)$ is given by entry A200753 in [11]. These numbers have apparently arisen before in various algebraic contexts, though we were unable to find other combinatorial interpretations in the literature.

\subsection{The cases 201 and 210}

THEOREM 3.8 If $n \geq 1$, then the sets $I \mathcal{S}_{n}(201)$ and $I \mathcal{S}_{n}(210)$ have the same cardinality.

Proof. By an $i$-occurrence of 201 or 210 in an inversion sequence $\lambda$, we will mean one in which the 2 corresponds to the actual letter $i$ in $\lambda$, and by an $(i, j)$-occurrence, where $i>j$, one in which the 2 and 1 correspond to the actual letters $i$ and $j$, respectively, in $\lambda$. We now construct a bijection $f$ between the sets $I \mathcal{S}_{n}(201)$ and $I \mathcal{S}_{n}(210)$ which systematically replaces $i$-occurrences of 210 with 201.

Let $\pi \in I \mathcal{S}_{n}(201)$. If $\pi$ contains no occurrences of 210 , then let $f(\pi)=\pi$. Otherwise, let $2 \leq$ $i_{1}<i_{2}<\cdots<i_{r} \leq n-1$ denote the set of distinct letters greater than one occurring in $\pi$. Consider the left-most occurrence of $i_{1}$ in $\pi_{0}=\pi$. Then any 0 's and 1's to the right of it must occur as a subsequence $S$ with all the 0's to the right of all the 1's. Reorder the 0's and 1's in $S$ so that all 1's occur to the right of all the 0 's. Note that this removes all $i_{1}$-occurrences of 210 and replaces them with $i_{1}$-occurrences of 201 . Let $\pi_{1}$ denote the resulting inversion sequence.

Now consider the left-most occurrence of the letter $i_{2}$ in $\pi_{1}$. Let $S$ denote the subsequence of $\pi_{1}$ consisting of all 0's, 1's, and $i_{1}$ 's occurring to the right of this $i_{2}$. Note that any $i_{1}$ 's in $S$ must occur prior to all 0's and 1's in $S$ or else there would have been an $\left(i_{2}, i_{1}\right)$-occurrence of 201 in $\pi$, which is 
impossible. Furthermore, each 1 occurs to the right of all 0 's in $S$, by the first step, since either the left-most $i_{2}$ of $\pi_{1}$ occurs to the right of the left-most $i_{1}$ or the left-most $i_{1}$ occurs to the right of the left-most $i_{2}$ but prior to any 0's and 1's in $S$. Reorder the letters of $S$ so that all $i_{1}$ 's come to the right of all 0's and 1's in $S$. Let $\pi_{2}$ denote the resulting inversion sequence. Note that any $i_{2}$-occurrences of 210 are removed in this step, while no $i_{1}$-occurrences of 210 are introduced, whence $\pi_{2}$ has no $i_{1}$ - or $i_{2}$-occurrences of 210 .

In general, consider the left-most occurrence of the letter $i_{j+1}$ in $\pi_{j}$, where $1 \leq j \leq r-1$. Let $S$ denote the subsequence of $\pi_{j}$ consisting of all 0's, 1's, and $i_{t}$ 's, $1 \leq t \leq j$, occurring to the right of this $i_{j+1}$. By induction, there are no $i_{t}$-occurrences of 210 for $1 \leq t \leq j$ in $\pi_{j}$. Furthermore, all $i_{j}$ 's occur before any smaller letters in $S$, for otherwise there would have been an $\left(i_{j+1}, i_{j}\right)$-occurrence of 201 in $\pi$ since no letters greater than or equal $i_{j}$ have been moved at this point. Reordering $S$ so that each $i_{j}$ occurs later than all other smaller letters of $S$ removes any $i_{j+1}$-occurrences of 210 , while introducing no smaller occurrences of 210 . The resulting sequence $\pi_{j+1}$ then has no $i_{t}$-occurrences of 210 for $1 \leq t \leq j+1$.

The procedure described above generates a sequence $\pi_{0}, \pi_{1}, \ldots, \pi_{r}$; note that $\pi_{r}$ has no $i_{t}$-occurrences of 210 for $1 \leq t \leq r$ and hence avoids 210. Let $f(\pi)=\pi_{r}$. For example, if $n=15$ and $\pi=$ $012322434875431 \in I \mathcal{S}_{15}(201)$, then

$$
\begin{aligned}
& \pi_{0}=01 \overline{2} 32243487543 \underline{1} \rightarrow \pi_{1}=012 \overline{3} \underline{22} 43487543 \underline{1} \rightarrow \pi_{2}=012312 \overline{4} \underline{3} 48754 \underline{32} \rightarrow \\
& \pi_{3}=01231242487 \overline{5} \underline{433} \rightarrow \pi_{4}=0123124248 \overline{7} \underline{5334} \rightarrow \pi_{5}=012312424 \overline{8} \underline{73345} \rightarrow \\
& \pi_{6}=012312424833457=f(\pi),
\end{aligned}
$$

where at each step $j$, the left-most occurrence of $i_{j+1}$ is overscored, while members of the corresponding subsequence $S$ are underscored. The mapping $f$ may be reversed by undoing each of the $r$ steps in the above procedure, starting with the last step and considering the left-most occurrence of the largest letter.

We seek the generating function for the sequence of cardinalities in Theorem 3.8. It will be easier to study the set $I \mathcal{S}_{n}(201)$, which we refine as follows. If $0 \leq m<t \leq n$, then let $\mathcal{C}_{n, m, t}$ denote the set of 201-avoiding inversion sequences of length $n$ whose largest letter is $m$ with the right-most occurrence of $m$ in position $t$. For example, the sequences 0123300 and 0011311 both belong to $\mathcal{C}_{7,3,5}$, whereas 0021332 and 0121312 do not. Let $c_{n, m, t}=\left|\mathcal{C}_{n, m, t}\right|$ if $0 \leq m<t \leq n$, and put $c_{n, m, t}=0$ otherwise. The array $c_{n, m, t}$ may be defined in the following manner.

LEMMA 3.9 If $1 \leq m<t \leq n$, then

$$
c_{n, m, t}=\sum_{i=m+1}^{t-1} c_{n-1, m, i}+\sum_{j=0}^{m-1} \sum_{i=j+1}^{t} c_{n-1, j, i}+\sum_{j=0}^{m-1} \sum_{i=1}^{n-t-1} c_{n-i-1, j, t},
$$

with $c_{n, 0, t}=\delta_{n, t}$ for $1 \leq t \leq n$.

Proof. The initial condition when $m=0$ is clear from the definitions. To show (19), first note that if the letter $m$ occurs more than once within a member of $\mathcal{C}_{n, m, t}$, then the right-most $m$ is extraneous concerning the avoidance of the pattern 201, and thus deletion of this $m$ results in a member of $\mathcal{C}_{n-1, m, i}$ for some $m+1 \leq i \leq t-1$. So suppose $m$ occurs once within $\pi \in \mathcal{C}_{n, m, t}$. Let $j$ denote the second largest 
letter of $\pi$. If the right-most occurrence of $j$ is in position $i$ for some $i \in\{j+1, j+2, \ldots, t-1\} \cup\{t+1\}$, then the $m$ is again extraneous concerning the avoidance of 201. Deletion of $m$ then results in a member of $\mathcal{C}_{n-1, j, i}$ for some $0 \leq j \leq m-1$ and $j+1 \leq i \leq t$. Finally, if the right-most occurrence of $j$ is in positions $t+2$ through $n$, then each letter between $m$ and the right-most $j$ must also be a $j$ in order to avoid 201. Deletion of $m$ as well as all $j$ 's to the right of $m$ except the last one results in a member of $\mathcal{C}_{n-i-1, j, t}$ for some $1 \leq i \leq n-t-1$ and $0 \leq j \leq m-1$. Note that the right-most $j$ slides to position $t$, upon deletion of some letters to its left, whence the third parameter of the resulting inversion sequence remains unchanged. Summing over $j$ and $i$ gives the third sum on the right-hand side of (19) and completes the proof.

Remark. We were unable to show directly the comparable recurrence to 19 for the pattern 210 , even though the bijection of Theorem 3.8 shows that the joint distribution for the statistics recording the largest letter and its right-most position is the same on the 210 avoidance class as it is on the 201 class.

If $n \geq 1$ and $0 \leq m<n$, then let

$$
c_{n, m}(v)=\sum_{t=m+1}^{n} c_{n, m, t} v^{t-m-1}
$$

If $n \geq 1$, let

$$
c_{n}(u, v)=\sum_{m=0}^{n-1} c_{n, m}(v) u^{m} .
$$

Define the generating function $h(x ; u, v)$ by

$$
h(x ; u, v)=\sum_{n \geq 1} c_{n}(u, v) x^{n} .
$$

Then $h(x ; u, v)$ is determined by the following functional equation.

THEOREM 3.10 We have

$$
h\left(x v_{+} ; u / v_{+}, 1\right)=\frac{v_{-}^{2}\left(1-v_{+}\right)}{v_{+}^{2}\left(1-v_{-}\right)} h\left(x v_{-} ; u / v_{-}, 1\right)+\frac{\left(v_{+}-v_{-}\right)\left(1-v_{+}\right)}{v_{+}^{2}},
$$

where $v_{ \pm}=v_{ \pm}(x, u)=\frac{u+1-x \pm \sqrt{(u+1-x)^{2}-4 u\left(1-x^{2}\right)}}{2\left(1-x^{2}\right)}$.

Proof. In order to convert recurrence (19) into functional equation form, we first replace $m$ with $m-1$ and subtract to obtain

$$
c_{n, m, t}-c_{n, m-1, t}=c_{n-1, m-1, t}+\sum_{i=m+1}^{t-1} c_{n-1, m, i}+\sum_{i=1}^{n-t-1} c_{n-i-1, m-1, t}, \quad m \geq 1 .
$$


Multiplying both sides of 21 by $v^{t-m-1}$ and summing over $m<t \leq n$ gives

$$
\begin{aligned}
c_{n, m}(v)- & \frac{1}{v}\left(c_{n, m-1}(v)-c_{n, m-1}(0)\right)=\frac{1}{v}\left(c_{n-1, m-1}(v)-c_{n-1, m-1}(0)\right) \\
& +\sum_{i=m+1}^{n-1} c_{n-1, m, i} \sum_{t=i+1}^{n} v^{t-m-1}+\frac{1}{v} \sum_{i=1}^{n-m-2} \sum_{t=m+1}^{n-i-1} c_{n-i-1, m-1, t} v^{t-m} \\
= & \frac{1}{v}\left(c_{n-1, m-1}(v)-c_{n-1, m-1}(0)\right)+\frac{v}{1-v}\left(c_{n-1, m}(v)-v^{n-m-1} c_{n-1, m}(1)\right) \\
& +\frac{1}{v} \sum_{i=1}^{n-m-2}\left(c_{n-i-1, m-1}(v)-c_{n-i-1, m-1}(0)\right)
\end{aligned}
$$

which may be rewritten as

$$
c_{n, m}(v)=\frac{v}{1-v}\left(c_{n-1, m}(v)-v^{n-m-1} c_{n-1, m}(1)\right)+\frac{1}{v} \sum_{i=m+1}^{n}\left(c_{i, m-1}(v)-c_{i, m-1}(0)\right)
$$

for $0 \leq m<n$. Multiplying 22 by $u^{m}$ and summing over $0 \leq m<n$ gives

$$
\begin{aligned}
c_{n}(u, v) & =\frac{v}{1-v}\left(c_{n-1}(u, v)-v^{n-1} c_{n-1}(u / v, 1)\right)+\frac{u}{v} \sum_{i=1}^{n} \sum_{m=1}^{i}\left(c_{i, m-1}(v)-c_{i, m-1}(0)\right) u^{m-1} \\
& =\frac{v}{1-v}\left(c_{n-1}(u, v)-v^{n-1} c_{n-1}(u / v, 1)\right)+\frac{u}{v} \sum_{i=2}^{n}\left(c_{i}(u, v)-c_{i}(u, 0)\right), \quad n \geq 2,
\end{aligned}
$$

with $c_{1}(u, v)=1$.

Multiplying both sides of (23) by $x^{n}$ and summing over $n \geq 2$, we obtain

$$
h(x ; u, v)-x=\frac{x v}{1-v}(h(x ; u, v)-h(x v ; u / v, 1))+\frac{u}{v(1-x)}(h(x ; u, v)-h(x ; u, 0)),
$$

which may be rewritten as

$$
\left(1-\frac{x v}{1-v}-\frac{u}{v(1-x)}\right) h(x ; u, v)=x-\frac{x v}{1-v} h(x v ; u / v, 1)-\frac{u}{v(1-x)} h(x ; u, 0) .
$$

Substituting $v=v_{+}$and $v_{-}$into (24), where $v_{ \pm}$are as defined above, yields

$$
\begin{aligned}
& x=\frac{x v_{+}}{1-v_{+}} h\left(x v_{+} ; u / v_{+}, 1\right)+\frac{u}{v_{+}(1-x)} h(x ; u, 0), \\
& x=\frac{x v_{-}}{1-v_{-}} h\left(x v_{-} ; u / v_{-}, 1\right)+\frac{u}{v_{-}(1-x)} h(x ; u, 0) .
\end{aligned}
$$

Eliminating $h(x ; u, 0)$ from the last two equations implies 20 .

Observe that $h(x ; u, 1)$ is the generating function which counts 201-avoiding inversion sequences according to the size of the largest letter. Though unable to complete a proof, we feel that the 
generating function $h(x ; u, 1)$ is not algebraic. One can, however, convert the functional equation 20 into a form which may then be iterated to obtain an expression for $h(x ; u, 1)$.

Let $\alpha=\alpha(x, u)$ and $\beta=\beta(x, u)$ be defined by

$$
\alpha(x, u)=\frac{1-u+\sqrt{(1-u)^{2}-4 x(1-x)(1-u)}}{2(1-x)}
$$

and

$$
\beta(x, u)=\frac{u\left(1-u-\sqrt{(1-u)^{2}-4 x(1-x)(1-u)}\right)}{2(1-u)} .
$$

Corollary 3.11 We have

$$
h(x ; u, 1)=\frac{w_{-}^{2}\left(1-w_{+}\right)}{w_{+}^{2}\left(1-w_{-}\right)} h\left(\alpha w_{-} ; \beta / w_{-}, 1\right)+\frac{\left(w_{+}-w_{-}\right)\left(1-w_{+}\right)}{w_{+}^{2}},
$$

where $w_{ \pm}=w_{ \pm}(x, u)=v_{ \pm}(\alpha, \beta)$.

Proof. To convert the first two arguments of $h$ on the left-hand side of 20 to $x$ and $u$, we need to solve the system $a v_{+}(a, b)=x$ and $b=v_{+}(a, b) u$ for $a$ and $b$, where $x$ and $u$ are given. To do so, first observe that $\frac{x}{a}$ is a root of the quadratic equation $\left(1-a^{2}\right) r^{2}+(a-1-b) r+b=0$, which implies

$$
\left(1-a^{2}\right) x^{2}+a(a-1-b) x+a^{2} b=0 .
$$

Since $a b=u x$, this equation is equivalent to

$$
\left(1-a^{2}\right) x^{2}+a^{2} x-a x-u x^{2}+a u x=0,
$$

which may be rewritten as

$$
(1-x) a^{2}+(u-1) a+x(1-u)=0 .
$$

Solving the last equation for $a$ gives $a=\alpha(x, u)$, and hence $b=\beta(x, u)$, where $\alpha$ and $\beta$ are as defined above. Replacing $x$ and $u$ with $\alpha$ and $\beta$ in 20 then gives (25).

\section{The case 120}

Though we are unable to ascertain an explicit formula for the number of 120 -avoiding inversion sequences of a given length, we can find a recurrence relating them to 021-avoiding $k$-ary words which have been previously studied (see, e.g., [1, 7]). We again refine the set whose cardinality is in question. If $n \geq 2$ and $0 \leq s<m<n$, then let $\mathcal{D}_{n, m, s}$ denote the set of 120-avoiding inversion sequences of length $n$ in which the largest letter is $m$ and the largest letter occurring to the left of the left-most occurrence of $m$ is $s$ (where we exclude from consideration the sequence consisting of all 0 's). Let $d_{n, m, s}=\left|\mathcal{D}_{n, m, s}\right|$. For example, if $n=4, m=2$, and $s=0$, then $d_{4,2,0}=4$, the enumerated sequences being 0020, 0021, 0022, and 0002 .

Let $\mathcal{B}_{n, k}$ denote the set of 120 -avoiding $k$-ary words of length $n$. If $n, k \geq 0$, then let $b_{n, k}=\left|\mathcal{B}_{n, k}\right|$, where $b_{0, k}=1$ if $k \geq 0$ and $b_{n, 0}=0$ if $n \geq 1$. The numbers $b_{n, k}$ are given by the formula (see [1])

$$
b_{n, k}=\sum_{j=1}^{n}(-1)^{n-j} C_{j}\left(\begin{array}{c}
j+k-1 \\
k-1
\end{array}\right)\left(\begin{array}{c}
j \\
n-j
\end{array}\right), \quad n, k \geq 1,
$$


where $C_{j}=\frac{1}{j+1}\left(\begin{array}{c}2 j \\ j\end{array}\right)$ denotes the $j$-th Catalan number.

The array of numbers $d_{n, m, s}$ may then be defined recursively as follows.

LEMMA 4.1 If $n \geq 3$, then

$$
d_{n, m, s}=\sum_{j=0}^{s-1} \sum_{i=1}^{n-m} d_{n-i, s, j} b_{i-1, m-s+1}, \quad 1 \leq s<m<n,
$$

and

$$
d_{n, m, 0}=\sum_{i=1}^{n-m} b_{i-1, m+1}, \quad 1 \leq m<n
$$

with $d_{2,1,0}=1$.

Proof. To show (26), let $\pi \in \mathcal{D}_{n, m, s}$ and assume that the left-most occurrence of the letter $m$ is in position $n-i+1$ for some $i \in[n-m]$. Then $\pi$ may be decomposed as $\pi=\pi^{\prime} m \alpha$, where $\pi^{\prime} \in \mathcal{D}_{n-i, s, j}$ for some $0 \leq j \leq s-1$ and $\alpha$ is a word of length $i-1$. Since $m$ is the largest letter of $\pi$ and $s \geq 1$, it follows that $\alpha$ contains no letters in $[s-1] \cup\{0\}$, for otherwise there would be an occurrence of 120 in $\pi$. Thus, $\alpha$ is a word in the alphabet $\{s, s+1, \ldots, m\}$ which avoids 120 since $\pi$ does. Note that no further restriction is required of $\alpha$ since all letters of $\alpha$ are greater than or equal all letters of $\pi^{\prime}$. Thus, $\alpha$ may be identified with a member of $\mathcal{B}_{i-1, m-s+1}$, which implies that there are $\sum_{j=0}^{s-1} \sum_{i=1}^{n-m} d_{n-i, s, j} b_{i-1, m-s+1}$ members of $\mathcal{D}_{n, m, s}$.

We now show (27). First observe that $\pi \in \mathcal{D}_{n, m, 0}$ may be decomposed as $\pi=0^{n-i} m \beta$, where $\beta$ is a word of length $i-1$ whose letters belong to the set $[m] \cup\{0\}$ and $1 \leq i \leq n-m$. Note that $\beta$ avoids the pattern 120 since $\pi$ does and that no further restriction is needed concerning $\beta$ since only 0 's and an $m$ occur to the left of $\beta$, neither of which can play the role of a 1 in an occurrence of 120 . Thus, $\beta$ is equivalent to a member of $\mathcal{B}_{i-1, m+1}$, and summing over $i$ gives 27 ).

Let $a_{j}$ be the sequence defined by

$$
\sum_{j \geq 0} a_{j} x^{j}=\frac{1}{2 x}\left(1-\sqrt{\frac{1-6 x+4 x^{2}}{1-2 x}}\right)=\frac{1-x}{1-2 x} C\left(\frac{x(1-x)}{1-2 x}\right),
$$

where $C(x)=\sum_{j \geq 0} C_{j} x^{j}$. Note that the sequence $a_{j}$ occurs as A059279 in [11].

Though we were unable to find $d_{n, m, s}$ in general, we do have the following partial result for when $s=0,1$.

PROPOSITION 4.2 If $n \geq 2$, then the number of 120-avoiding inversion sequences of length $n$ having $s$ value zero is given by

$$
\sum_{j=1}^{n-1}(n-j) C_{j}
$$

and the number of sequences having s value one is given by

$$
\sum_{j=1}^{n-2}\left(2^{n-j-1}-1\right) a_{j}-\sum_{j=1}^{n-2}(n-j-1) C_{j}
$$

where $a_{j}$ is as defined above. 
Proof. If $n \geq 2$ and $1 \leq m \leq n-1$, then let $d_{n, m}(v)=\sum_{s=0}^{m-1} d_{n, m, s} v^{s}$ and $d_{n}(u, v)=\sum_{m=1}^{n-1} d_{n, m}(v) u^{m-1}$. Define the generating functions $D(x ; u, v)$ and $B(x, y)$ by

$$
D(x ; u, v)=\sum_{n \geq 2} d_{n}(u, v) x^{n}
$$

and

$$
B(x, y)=\sum_{n \geq 0} \sum_{k \geq 0} b_{n, k} x^{n} y^{k}
$$

Note that 120 -avoiding $k$-ary words are equivalent to 021-avoiding words, by reversal. Recalling the well-known generating function formula for the latter (see [7]), we have

$$
B(x, y)=1+\frac{y}{2 x(1-x)}\left(1-\sqrt{\frac{(1-2 x)^{2}-y}{1-y}}\right) .
$$

By 27 and (28), we then have

$$
\begin{aligned}
D(x ; u, 0) & =\sum_{n \geq 2} x^{n} \sum_{m=1}^{n-1} d_{n, m, 0} u^{m-1}=\sum_{n \geq 2} x^{n} \sum_{m=1}^{n-1} \sum_{i=1}^{n-m} b_{i-1, m+1} u^{m-1} \\
& =\sum_{m \geq 1} \sum_{i \geq 0} b_{i, m+1} u^{m-1} \sum_{n \geq m+i+1} x^{n}=\frac{1}{u^{2}(1-x)} \sum_{m \geq 1} \sum_{i \geq 0} b_{i, m+1} x^{i}(x u)^{m+1} \\
& =\frac{1}{u^{2}(1-x)}\left(B(x, x u)-1-\frac{x u}{1-x}\right) \\
& =\frac{1}{2 u(1-x)^{2}}\left(1-\sqrt{\frac{(1-2 x)^{2}-x u}{1-x u}}\right)-\frac{x}{u(1-x)^{2}} .
\end{aligned}
$$

This implies

$$
\begin{aligned}
D(x ; 1,0) & =\frac{1}{2(1-x)^{2}}(1-\sqrt{1-4 x})-\frac{x}{(1-x)^{2}}=\frac{x}{(1-x)^{2}} \sum_{j \geq 1} C_{j} x^{j} \\
& =\sum_{i \geq 1} i x^{i} \sum_{j \geq 1} C_{j} x^{j} .
\end{aligned}
$$

Thus, the coefficient of $x^{n}$ in $D(x ; 1,0)$ is given by $\sum_{j=1}^{n-1}(n-j) C_{j}$, which implies the first statement.

To show the second, we substitute $d_{n, 1,0}=2^{n-1}-1$ into 26 to get

$$
d_{n, m, 1}=\sum_{i=1}^{n-m}\left(2^{n-i-1}-1\right) b_{i-1, m}, \quad 1 \leq s<m<n .
$$


Proceeding as in the prior case, we find

$$
\begin{aligned}
\sum_{n \geq 3} x^{n} \sum_{m=2}^{n-1} d_{n, m, 1} & =\sum_{n \geq 3} x^{n} \sum_{m=2}^{n-1} \sum_{i=1}^{n-m}\left(2^{n-i-1}-1\right) b_{i-1, m} \\
& =\frac{x}{2(1-2 x)}\left(B(x, 2 x)-1-\frac{2 x}{1-x}\right)-\frac{x}{1-x}\left(B(x, x)-1-\frac{x}{1-x}\right) \\
& =\frac{x^{2}}{(1-x)(1-2 x)}\left(\frac{1-\sqrt{\frac{1-6 x+4 x^{2}}{1-2 x}}}{2 x}-1\right)-\frac{x^{2}}{(1-x)^{2}}\left(\frac{1-\sqrt{1-4 x}}{2 x}-1\right),
\end{aligned}
$$

and extracting the coefficient of $x^{n}$ gives the second statement.

Remark. Comparable formulas for other small values of $s$ may be found recursively using (26). In the first formula above, note that $\sum_{j=1}^{n-1}(n-j) C_{j}=b_{n}-n$, where $\left(b_{n}\right)_{n \geq 1}=(1,3,7,16,39,104, \ldots)$ is A014140 in [11].

\section{Conclusion}

We have provided explicit formulas and/or generating functions for the number of inversion sequences avoiding a single member of $\mathcal{S}_{3}$. In three of the cases, we have made use of the kernel method to ascertain the generating function. In the cases of 012 and 021 , it would be interesting to find direct combinatorial proofs in light of Theorems 2.1 and 3.3. We also seek a closed form expression for the cardinality of $\left|I \mathcal{S}_{n}(120)\right|$ or its generating function. Concerning future research directions, one might consider the problem of avoiding two or more patterns in $\mathcal{S}_{3}$, though here the results seem to be somewhat sparse. More promising are results concerning the avoidance of a single permutation pattern of length greater than three or those concerning the avoidance of a generalized pattern in which one or more of the letters are required to be adjacent, in particular those of type $x y-z$ or $x-y z$. One could also consider the avoidance of a pattern having one or more repeated letters.

\section{References}

[1] A. Burstein, Enumeration of Words with Forbidden Patterns, Ph.D. thesis, University of Pennsylvania, 1998.

[2] D. Callan, Pattern avoidance in "flattened" partitions, Discrete Math., 309 (2009) 41874191.

[3] W. Y. C. Chen, A. J. X. Guo, P. L. Guo, H. H. Y. Huang And T. Y. H. Liu, s-inversion sequences and P-partitions of type B, at http://arxiv.org/abs/1310.5313.

[4] Q. H. Hou And T. Mansour, Kernel method and systems of functional equations with several conditions, J. Comput. Appl. Math., 235 (2011) 1205-1212.

[5] S. Kitaev, Patterns in Permutations and Words, Springer, 2011. 
[6] D. E. Knuth, The Art of Computer Programming, Volumes 1 and 3, Addison-Wesley, Reading, MA 1968, 1973.

[7] T. Mansour, Restricted 132-avoiding k-ary words, Chebyshev polynomials, and continued fractions, Adv. in Appl. Math., 36 (2006) 175-193.

[8] T. Mansour and M. Shattuck, Pattern avoidance in flattened permutations, Pure Math. Appl. (PU.M.A.), 22 (2011) 75-86.

[9] C. D. Savage and M. J. Schuster, Ehrhart series of lecture hall polytopes and Eulerian polynomials for inversion sequences, J. Combin. Theory Ser. A, 119 (2012) 850-870.

[10] R. Simion and F. W. Schmidt, Restricted permutations, European J. Combin., 6 (1985) $383-406$.

[11] N. J. A. Sloane, The On-Line Encyclopedia of Integer Sequences, at http://oeis.org, 2010.

[12] R. P. Stanley, Enumerative Combinatorics, Vol. I, Cambridge University Press, 1997.

[13] D. Zeilberger, Enumeration schemes, and more importantly, their automatic generation, Ann. Comb., 2 (1998) 185-195. 